\title{
High frequency AC electrosprays: mechanisms and applications
}

\author{
L. Y. Yeo ${ }^{1} \&$ H.-C. Chang ${ }^{2}$ \\ ${ }^{1}$ Micro/Nanophysics Research Laboratory, \\ Department of Mechanical Engineering, Monash University, \\ Clayton, Australia \\ ${ }^{2}$ Department of Chemical and Biomolecular Engineering, \\ University of Notre Dame, Notre Dame, U.S.A.
}

\begin{abstract}
We have recently discovered a new electrospray phenomenon using high frequency AC electric fields beyond $10 \mathrm{kHz}$ which behaves very distinctly from its DC electrospray counterpart. Experimental and theoretical findings have demonstrated that plasma charging plays a significant role in the electrohydrodynamically-driven interfacial dynamics of the electrospray. These findings show that Faradaic generation of interfacial plasma polarized layers can produce a net normal Maxwell stress that is responsible for the generation of aerosol drops. Experimental results of this plasma polarization phenomenon are presented and scaling theory is employed to elucidate the underlying plasma mechanism responsible for the phenomenon. The AC electrospray is capable of producing micron and sub-micron electroneutral drops or polymeric fibers and is a promising rapid and portable technology for drug encapsulation or biomaterials synthesis for controlled release respiratory drug delivery, wound care therapy or tissue/orthopaedic engineering.
\end{abstract}

\section{Introduction}

DC electric fields have mainly been used in electrohydrodynamic atomization (otherwise known as electrospraying) [1]. Several DC electrospray modes have been observed [2], the predominant mode being the cone-jet mode in which the liquids meniscus assumes the shape of a sharp cone with half angle approximately equal to the Taylor angle of $49.3^{\circ}$, obtained by balancing the electrostatic and capillary pressures acting on a conical surface for ideal, static equilibrium conditions [3]. 

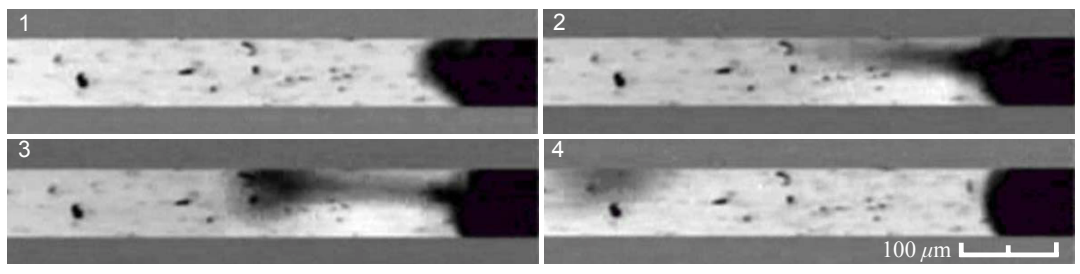

Figure 1: Image sequences at 6000 frames/s taken $300 \mu \mathrm{s}$ apart illustrating microjet formation and subsequent drop detachment at $f=15 \mathrm{kHz}$ and $V=4000 \mathrm{~V}$.

Where AC fields have been utilized, these have been limited to low frequencies $(<1 \mathrm{kHz})$ such that the electrospray behavior does not significantly deviate from that of its DC counterpart [4]. Recently, the authors and their collaborators reported on a new high frequency (10-280 kHz) AC electrospray [5]. The drops generated were observed to be of larger dimensions $(\sim 10 \mu \mathrm{m})$ and were shown to carry no net charge, in contrast to that produced by DC electrosprays. In addition, none of the DC modes were observed and the drops were not ejected from the steady, well-defined Taylor cone characteristic of the stable DC modes. Instead, the drops are ejected from a round meniscus or from a peculiar microjet that protrudes intermittently from the meniscus (Fig. 1). Unlike the DC cone-jets, the microjets do not emerge from a Taylor cone and are much larger in size. In this paper, we briefly present evidence from experiments and scaling arguments to support an interfacial plasma polarization mechanism responsible for the dynamics of the AC electrospray. In addition, we also show the potential of the AC electrospray as a viable vehicle for drug encapsulation and biomaterials synthesis.

\section{DC Taylor cone}

In DC electrospraying, the absence of an external periodic forcing allows sufficient time for charge separation to take place in the liquid meniscus upon application of an electric field between the tip of the micro-needle from which the liquid meniscus emanates and a grounded electrode placed at a distance. Tangential ion conduction then occurs along the electric double layer formed at the interface thus resulting in co-ion accumulation and hence a singular electric field at the meniscus tip [6]. The repulsion between the co-ions at the meniscus tip then results in Coulombic fission wherein a thin liquid jet emanates from the tip once the repulsive force exceeds the surface force. The co-ion accumulation at the meniscus tip is also the reason why the drops produced as a result of various instabilities suffered by the jet carry a net charge.

For a perfectly conducting liquid, Taylor [3], by considering the static equilibrium balance between the capillary and Maxwell stresses, showed that a conical meniscus with a half angle of $49.3^{\circ}$ is produced. Due to the assumption of the perfect conducting limit, the drop is at constant potential and hence the gas-phase 
electric field is predominantly normal at the meniscus interface. It can then be shown that the normal gas phase electric field $E_{n, g}$ scales as $1 / \sqrt{R}$, where $R$ is the meniscus radius, and hence the Maxwell pressure, $p_{\mathrm{M}} \sim E_{n, g}^{2}$ scales as $1 / R$. $p_{\mathrm{M}}$ therefore exactly balances the azimuthal capillary pressure $p_{\mathrm{C}} \sim \gamma / R$, where $\gamma$ is the interfacial tension, for all values of $R$. This exact balance, and absence of a length scale selection, is responsible for the formation of a static Taylor cone.

Taylor's perfectly conducting limit was extended to allow for the effect of finite liquid conductivities by Li et al. [7] and Stone et al. [8]. Both of these studies showed that in these cases, it is the tangential electric field within the slender conical liquid meniscus that dominates. Nevertheless, it can be shown that the tangential liquid phase electric field $E_{t, l}$ also scales as $1 / R$ and thus an exact balance between the Maxwell stress $p_{\mathrm{M}} \sim E_{t, l}^{2}$ and the capillary stress $p_{\mathrm{C}} \sim \gamma / R$ is again obtained, giving rise to a conical-like structure. The cone angle, however, depends crucially on the liquid to gas permittivity ratio $\beta \equiv \epsilon_{l} / \epsilon_{g}$, and the Taylor angle is recovered in the perfectly conducting limit as $\beta \rightarrow \infty$.

\section{AC microjet}

\subsection{Plasma polarization mechanism}

In AC electrosprays, the high frequency periodic forcing does not permit sufficient time for charge separation and hence co-ion accumulation at the meniscus tip. As a result, the generated drops are electroneutral. Moreover, the absence of tangential ion conduction also stipulates a weaker liquid phase tangential electric field, consistent with our earlier experimental findings in which the AC electrospray behavior was found to be insensitive to liquid conductitivity [5]. This passive role of the liquid phase is compounded by the formation of a thin, highly-conducting, permanent negatively charged plasma polarization layer around the liquid meniscus that gives rise to a dominant normal gas phase electric field in one AC half cycle. By imposing a flow of inert gases known to catalyze plasma ionization along the liquid meniscus, Lastochkin \& Chang [9] showed that the critical voltage for drop ejection in AC electrospraying can be substantially lowered.

When ejected drops are passed through a set of parallel capacitor plates, their continuous attraction to the positive plate despite periodic reversal of the AC polarity demonstrates the existence of a permanent negatively charged plasma cloud surrounding the drop [6]. This negative charge does not originate within the drop due to the lack of time for charge separation in the liquid, as discussed above. In addition, the possibility of the drop carrying charge is ruled out since the drop ejection time, typically $10^{-3} \mathrm{~s}$, is much greater than applied AC forcing period thus allowing any charge within the drop to essentially equilibrate during the ejection event. In addition, liquid conductivity again was not observed to influence the degree of drop deflection between the plates.

Given the passivity of the liquid phase, we postulate that the plasma cloud arises due to liquid vaporization from the meniscus and its subsequent ionization to produce negative ions. This is evidenced by the observation that aerosol gen- 


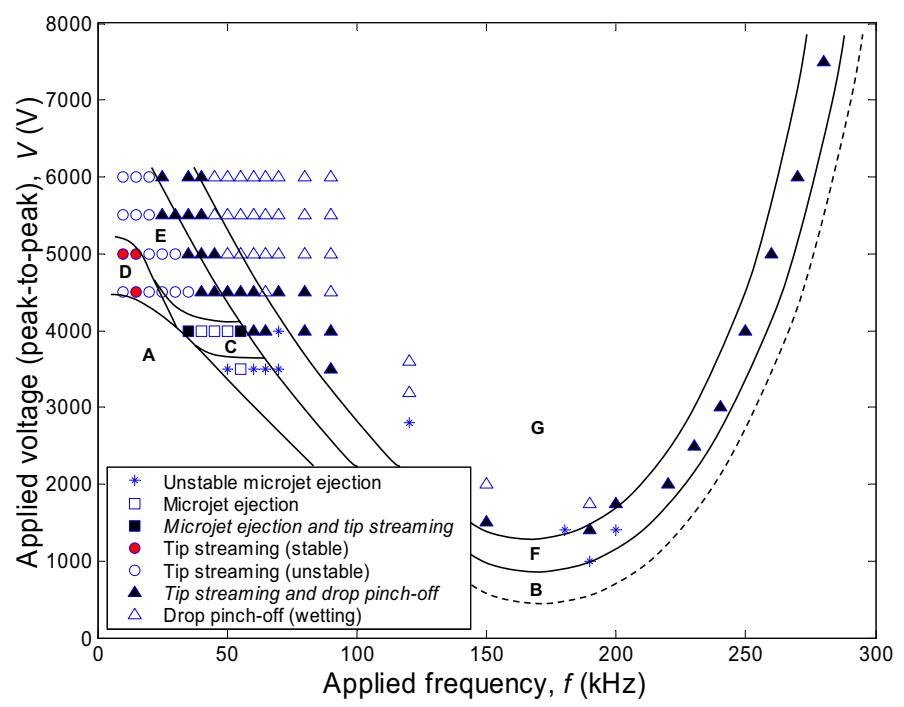

Figure 2: AC electrospray behavior indicating the optimum frequency at which the minimum in the critical voltage occurs [5]. The various spray modes are also shown: A-Capillary dominant regime (no drop ejection), B-Unstable microjet ejection, C-Microjet ejection with/without tip streaming, D-Stable tip streaming, E-Unstable tip streaming, F-Tip streaming with drop pinch-off (onset of wetting), and $\mathrm{G}$-Drop pinch-off and wetting.

eration in the AC electrospray only occurs when the working liquid possesses a sufficiently high volatility (e.g. alcohols) and when the applied voltage exceeds a threshold voltage associated with the ionization potential [6]. Moreover, the Vshaped frequency dependence of the electrospray indicating a frequency optimum ( $\sim 165 \mathrm{kHz}$ ) at which the critical voltage is at its minimum (Fig. 2 [5]) lends further support to the postulated plasma polarization mechanism. The gas phase ions generated around the meniscus will diffuse away unless the frequency is sufficiently high such that the ionization rate in one half AC cycle exceeds the dispersion rate. On the contrary, at extremely high frequencies, there is insufficient time for plasma ions to be generated through a Faradaic reaction mechanism. As such, an optimum frequency is anticipated at which maximum plasma polarization occurs, which thus gives rise to maximum enhancement of the local normal Maxwell field at the meniscus interface.

Other possibilities exist that can explain the existence of the permanent charge cloud [6]. Nevertheless, we will not concern ourselves with exactly how the plasma layer arises but rather the effects of its existence. In the cathodic half cycle, when the meniscus and micro-needle have the same polarity as the plasma cloud which forms a thin highly conducting layer around the meniscus, the local gas phase 


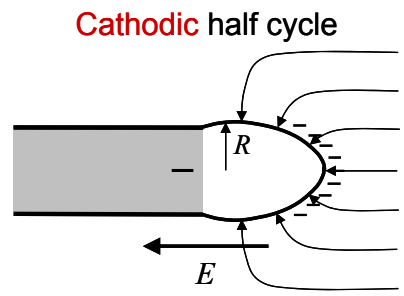

(a)
Anodic half cycle

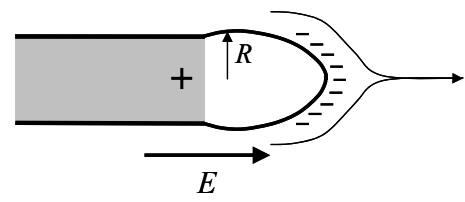

(b)

Figure 3: Postulated plasma polarization mechanism. (a) In the cathodic half cycle, the plasma cloud effectively enhances the local normal gas phase electric field. (b) In the anodic half cycle, the plasma cloud effectively screens the external field to produce a weak tangential gas phase electric field.

normal electric field at the interface is significantly enhanced, as illustrated in Fig. 3(a). This is because the meniscus and the plasma layer both resemble constant potential bodies in which the interfacial field is predominantly normal. In the anodic half cycle, however, whilst plasma is not generated, the plasma layer remains as there is insufficient time for its dispersion. The plasma layer is now oppositely charged to the meniscus and micro-needle and hence effectively screens the external field such that a weak tangential gas phase field arises, as depicted in Fig. 3(b). Consequently, it is the enhanced normal gas phase field that dominates and hence, averaged over many cycles, produces a net Maxwell stress that is responsible for the meniscus dynamics observed. Henceforth, we shall therefore restrict our analysis to this dominant normal gas phase field.

Given that both the meniscus and plasma layer resemble constant potential bodies, the solution of the Laplace equation governing the gas phase electrostatics gives rise to a specific scaling for the normal gas phase electric field $E_{n, g}$ at the interface. At this juncture, we will assume an arbitrary axisymmetric meniscus shape and not preclude the existence of a conical geometry. Thus, for a sharp conical meniscus, it can be shown from spheroidal harmonics that $E_{n, g} \sim 1 / \sqrt{R}$ whereas for more slender bodies such as an elongated ellipsoid or cylinder, $E_{n, g} \sim$ $1 / R$ [6]. Nevertheless, we note that at the meniscus tip, as $R \rightarrow 0$, the more singular $1 / R$ scaling for a slender geometry dominates. It then follows that the Maxwell pressure $p_{\mathrm{M}} \sim E_{n, g}^{2}$ scales as $1 / R^{2}$ and hence an exact balance with the azimuthal capillary pressure $p_{\mathrm{C}} \sim \gamma / R$ is only possible for one specific value of $R$. This length scale selection therefore excludes the possibility of a conical geometry and instead suggests that the meniscus is stretched to a more elongated cylinder-like geometry such as that of a microjet (regimes B and C in Fig. 2), as depicted in Fig. 1. This scaling therefore explains the appearance of a non-steady microjet instead of a steady Taylor cone in AC electrosprays. 


\subsection{Dynamic microjet simulation}

The role of the Maxwell pressure resulting from a normal gas phase interfacial electric field that scales as $1 / R$ in elongating the liquid meniscus into a cylindrical microjet structure can also be verified through a dynamic simulation in which the equations governing the coupled interactions between the hydrodynamics and electrodynamics are solved simultaneously in the longwave limit in axisymmetric polar coordinates $(r, 0, z)$. The fundamental assumption here is that the axial length scale $L$ of the liquid meniscus and resulting jet is large compared to the radial length scale $R_{0}$, i.e. $\epsilon \equiv R_{0} / L \ll 1$. We adopting the following transformations,

$$
\begin{gathered}
(r, R) \rightarrow R_{0}\left(r, R_{0}\right), \quad z \rightarrow L z, p \rightarrow \frac{\gamma}{R_{0}} p, \\
u \rightarrow \frac{U u}{\epsilon}, \quad v \rightarrow U v, \quad t \rightarrow \frac{R_{0} t}{U}, \quad \phi \rightarrow V \phi,
\end{gathered}
$$

where $u$ and $v$ are the axial and radial velocities, $p$ is the pressure and $t$ is the time. $U \equiv \gamma / \mu$ is the characteristic velocity scale with $\mu$ being the liquid viscosity and $V$ the applied potential.

Substituting the above transformations into the axisymmetric continuity and Navier-Stokes equations governing mass and momentum conservation in the liquid jet, together with the relevant interfacial boundary conditions (normal stress jump, tangential stress continuity and kinematic boundary conditions), and, exploiting the small value of $\epsilon$ by expanding the solutions for all variables in powers of $\epsilon$, we then arrive at the following leading order evolution equations for the axial velocity and jet radius: $[10,6]$

$$
\begin{gathered}
\operatorname{Re}\left(u^{(0)_{t}}+u^{(0)} u_{z}^{(0)}\right)=-\left(\frac{1}{R^{(0)}}\right)_{z}+B p_{\mathrm{M}_{z}}^{(0)}+\frac{6 R_{z}^{(0)} u_{z}^{(0)}}{R^{(0)}}+3 u_{z z}^{(0)}, \\
R_{t}^{(0)}+u^{(0)} R_{z}^{(0)}+\frac{R^{(0)} u_{z}^{(0)}}{2}=0
\end{gathered}
$$

where $\operatorname{Re} \equiv \epsilon^{2} \rho U R_{0} / \mu$ is the Reynolds number, demoted to higher order, with $\rho$ being the liquid density. The subscripts indicate partial derivatives in space and time. $B \equiv \varepsilon_{g} E_{\infty}^{2} R_{0} / 2 \gamma$ is the Maxwell Bond number with $E_{\infty}$ being the applied electric field. The leading order Maxwell pressure $p_{M}^{(0)}$ is given by a composite formulation involving the Maxwell stress for a spherical cap at the meniscus tip and the Maxwell stress for a cylindrical geometry (with the same scaling as described above), connected by a weighting function [6]:

$$
\begin{aligned}
p_{\mathrm{M}}^{(0)}= & {\left[1-\exp \left(-z / R_{s}\right)\right]\left(\frac{L z}{R_{0} R^{(0)}}\right)^{2} } \\
& +\exp \left(-z / R_{s}\right)\left\{\cos \alpha\left[1+2\left(\frac{R_{s} \sin \alpha}{R^{(0)}}\right)^{3}\right]\right\}^{2},
\end{aligned}
$$




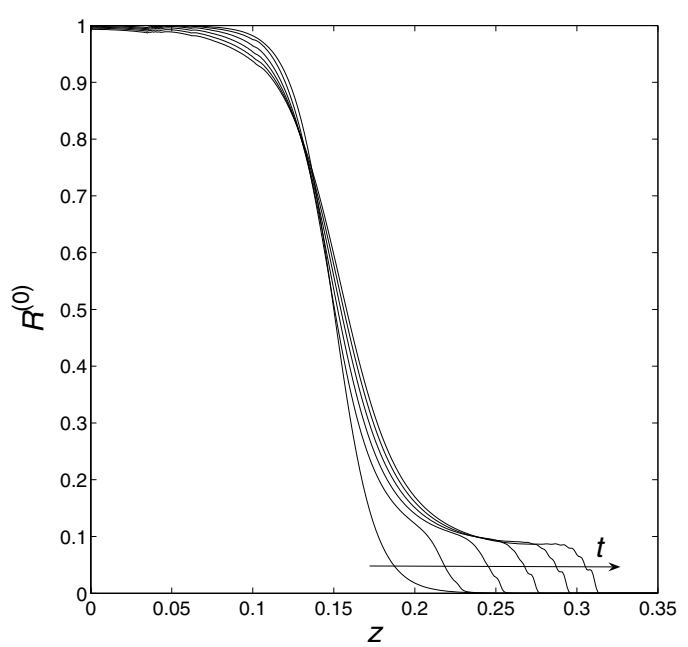

Figure 4: Typical spatio-temporal evolution profile of the meniscus interface for 5 equal time steps up to $t=0.4$ with $B=10$ and $\mathrm{Re}=100$. The initial profile is observed to elongate into a long slender microjet due to the action of the normal Maxwell stress at the interface.

with $\alpha=\tan ^{-1}\left(R / R_{s}-z\right)$ where $R_{s}$ is the radius of curvature of the spherical meniscus tip.

Equations (2) and (3) with Eq. (5) are solved numerically using the Method of Lines $[11,12]$ subject to the following initial conditions:

$$
R^{(0)}=0.5\left[1-\tanh \left(\frac{z-0.15}{0.025}\right)\right], \quad \text { and, } \quad u^{(0)}=0,
$$

and boundary conditions $R^{(0)}=1$ and $u^{(0)}=0$ at $z=0$, and, $R^{(0)} \rightarrow 0$ and $u^{(0)}=0$ as $z \rightarrow \infty$. A typical spatio-temporal evolution profile is illustrated in Fig. 4 in which the an axial pressure gradient resulting from the interfacial distribution of the normal Maxwell stress with $1 / R^{2}$ scaling along the meniscus is observed to elongate the initially rounded meniscus and pull out a slender microjet close to that observed in Fig. 1. After a short transient, the microjet is observed to propagate forward at roughly constant velocity whilst maintaining an approximately constant radius.

\section{AC electrospray applications}

The main advantages of employing AC fields over DC in electrospraying are the inherent safety of employing high frequency AC, the lower critical voltages required and the electroneutrality of the ejected drops. The electroneutral drops stipulate negligible current and hence a low power requirement, which enables the 
AC electrospray to be miniaturized for the design of small portable devices. Moreover, the use of high frequency AC ensures safe use, particularly in the case of consumer applications.
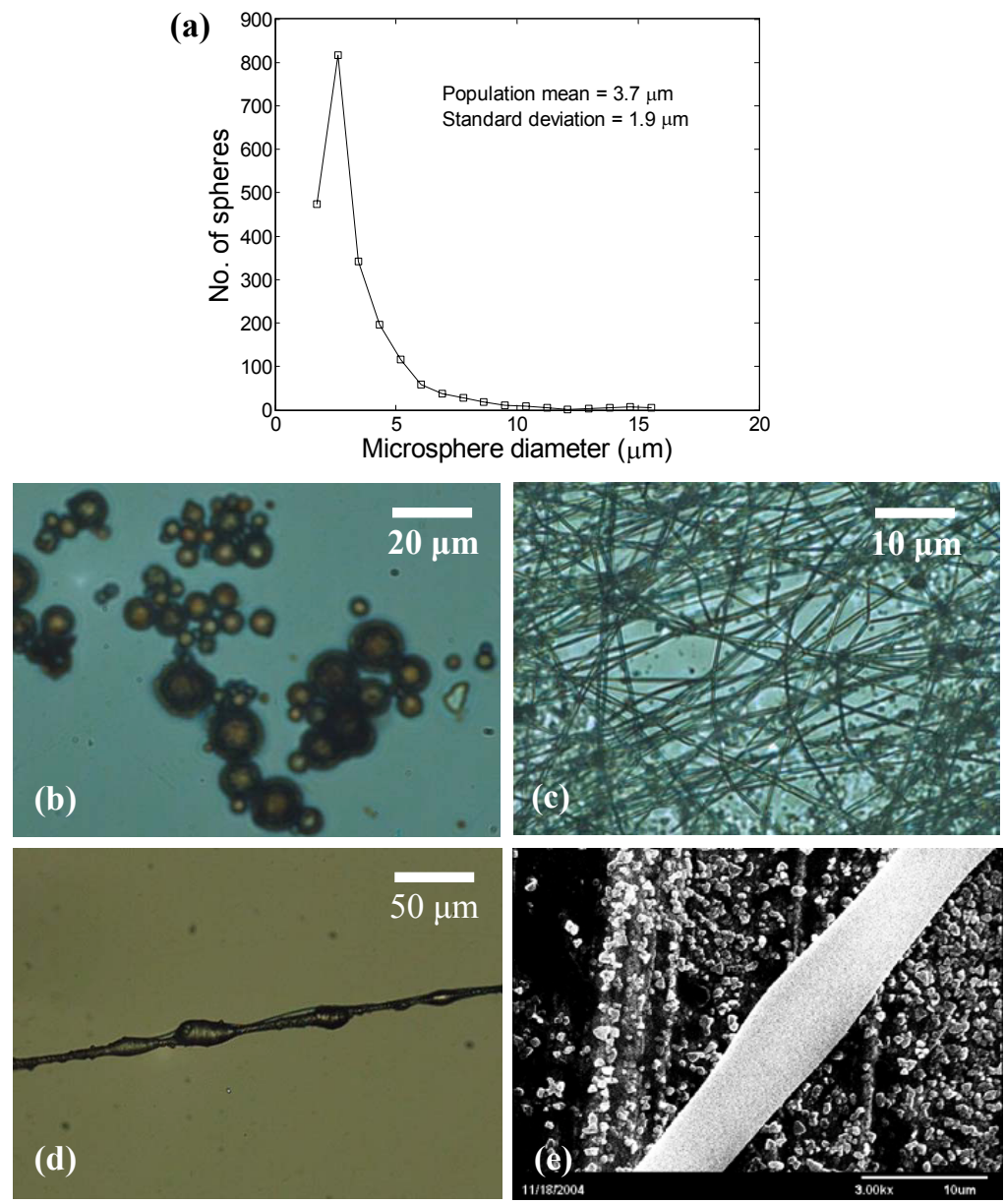

Figure 5: Synthesis of microparticles and microfibers from poly(lactic acid) using the AC electrospray. (a) Particle size distribution. (b) Water encapsulated within poly(lactic acid) microspheres. (c) Two-dimensional scaffolding network of micron sized poly(lactic acid) fibers with adjustable pore sizes. (d) 10 micron single compound poly(lactic acid) fiber. (e) Scanning electron microscope image of a micron sized fiber overlaid on a background of $100 \mathrm{~nm}$ poly(lactic acid) particles.

The stability of the ejected drops from Coulombic fission enables the synthesis of drug/vaccine encapsulated micron-sized spherical particles and fibers consist- 
ing of biodegradable polymeric materials. Yeo et al. [13] discusses the relative merits of using AC fields over DC electrospraying and other conventional synthesis methods, particularly for the rapid mass production of bioscaffolds for tissue/orthopaedic engineering or for miniaturized consumer drug delivery or wound care therapy devices. In particular, the electroneutral drops prevent surface adsorption and compound ionization for in vivo applications such as drug delivery. Figure 5 shows examples of water encapsulated microparticles and microfibers made from poly(lactic acid) using the AC electrospray. Peculiarly, nanoparticles are also observed using scanning electron microscopy, as shown in Fig. 5(e), although further characterization of such is needed [13].

\section{References}

[1] Grace, J.M. \& Marijnissen, J.C.M., A review of liquid atomization by electrical means. J. Aerosol Sci., 25, pp. 1005-1019, 1994.

[2] Clopeau, M. \& Prunet-Foch, B. Electrohydrodynamical spraying functioning modes: a critical review. J. Aerosol Sci., 25, pp. 1021-1036, 1994.

[3] Taylor, G. I., Disintegration of water drops in an electric field. Proc. R. Soc. Lond. A 280 (1964) 383-397.

[4] Borra, J.P., Tombette Y. \& Ehouarn, P., Influence of electric field profile and polarity on the mode of EHDA related to electric discharge regimes. J. Aerosol Sci., 30, pp. 913-925, 1999.

[5] Yeo, L.Y., Lastochkin, D., Wang, S.-C. \& Chang, H.-C., A new AC electrospray mechanism by Maxwell-Wagner polarization and capillary resonance. Phys. Rev. Lett., 92, 133902, 2004.

[6] Maheshwari, S., Yeo, L.Y. \& Chang, H.-C., High frequency AC electrosprays. Submitted to Phys. Fluids.

[7] Li, H., Halsey, T.C. \& Lobkovsky, A., Singular shape of a fluid drop in an electric or magnetic field. Europhys. Lett., 27, pp. 575-580, 1994.

[8] Stone, H.A., Lister, J.R. \& Brenner, M.P., Drops with conical ends in electric and magnetic fields. Proc. Roy. Soc. Lond. A, 455, pp. 329-347, 1999.

[9] Lastochkin, D. \& Chang, H.-C., A high frequency electrospray driven by gas volume charges. J. Appl. Phys., 97, 123309, 2005.

[10] Eggers, J., Universal Pinching of 3D axisymmetric free-surface flows. Phys. Rev. Lett., 71, pp. 3458-3460, 1993.

[11] Schiesser, W.E., The Numerical Method of Lines, Academic: San Diego, 1991.

[12] Yeo, L.Y., Matar, O.K., Perez de Ortiz, E.S. \& Hewitt, G.F., The dynamics of Marangoni-driven local film drainage between two drops. J. Colloid Interface Sci., 241, pp. 233-247, 2001.

[13] Yeo, L.Y., Gagnon, Z. \& Chang, H.-C., AC electrospray biomaterials synthesis. Biomaterials, 26, pp. 6122-6128, 2005. 